\title{
Significance of Size of Lymph Node Metastasis on Postsurgical Stimulated Thyroglobulin Levels After Prophylactic Unilateral Central Neck Dissection in Papillary Thyroid Carcinoma
}

\author{
Brian Hung-Hin Lang, MS' ${ }^{1}$, Alex H. Tang, $\mathrm{MBBS}^{2}$, Kai Pun Wong, MBBS ${ }^{1}$, Tony W. Shek, MBBS ${ }^{2}$, \\ Koon Yat Wan, MBBS ${ }^{3}$, and Chung-Yau Lo, MS $^{1}$ \\ ${ }^{1}$ Division of Endocrine Surgery, Department of Surgery, The University of Hong Kong, Hong Kong SAR, China; \\ ${ }^{2}$ Department of Anatomical Pathology, Queen Mary Hospital, Hong Kong SAR, China; ${ }^{3}$ Department of Clinical Oncology, \\ The University of Hong Kong, Hong Kong SAR, China
}

\begin{abstract}
Background. The prognostic significance of size of central lymph node metastasis (CLNM) in papillary thyroid carcinoma (PTC) remains unknown. Because postsurgical detectable stimulated thyroglobulin (DsTg) after radioiodine ablation may imply persistent or recurrent disease, we evaluated the association between size of CLNM and rate of DsTg in patients with PTC who underwent unilateral prophylactic central neck dissection.

Methods. To be eligible for analysis, the prophylactic central neck dissection specimen with $<3$ central lymph nodes (CLNs) or size of CLNM $\geq 1 \mathrm{~cm}$ as measured under the microscope was excluded. Of 132 specimens, 89 (67.4\%) were eligible. Forty patients $(44.9 \%)$ had no metastasis or pN0, $20(22.5 \%)$ had micrometastasis $(<2 \mathrm{~mm})$ or pN1mic and $29(32.6 \%)$ had macrometastasis $(\geq 2 \mathrm{~mm})$ or pN1mac. Postablation sTg level was measured 9 months after surgery. A multivariable analysis was conducted to identify independent factors for postablation DsTg.

Results. Larger-sized CLNM correlated significantly with younger age $(p=0.028)$, greater number of CLN retrieved $(p=0.016)$, greater number of metastatic CLN excised $(p<0.001)$, higher metastatic CLN ratio $(p=0.006)$ and postablation sTg level $(p=0.012)$. In the multivariable analysis, after adjusting for tumor size and metastatic CLN ratio, size of CLNM was an independent predictor of postablation DsTg (odds ratio $1.56,95 \%$ confidence
\end{abstract}

(C) The Author(s) 2012. This article is published with open access

at Springerlink.com

First Received: 21 January 2012;

Published Online: 8 May 2012

B. H.-H. Lang, MS

e-mail: blang@hku.hk interval $1.09-2.24, p=0.015)$. Relative to $\mathrm{pN} 0$, the odds ratios for postablation DsTg in $\mathrm{pN} 1 \mathrm{mic}$ and $\mathrm{pN} 1 \mathrm{mac}$ were 2.53 (95\% confidence interval 0.35-19.00, $p=0.351$ ) and 5.81 (95\% confidence interval $1.22-27.70, p=0.027$ ), respectively.

Conclusions. Size of CLNM was an independent factor for DsTg 9 months after surgery. Patients with pN1mac were almost 6 times more likely to have postablation DsTg than those with pN0 or pN1mic.

Papillary thyroid carcinoma (PTC) is the most common type of differentiated thyroid carcinoma, and its ageadjusted incidence has doubled in the last 25 years. ${ }^{1}$ Despite its relatively good prognosis, with a cancer-specific survival above $90 \%$, locoregional recurrence (LRR) is common. ${ }^{2}$ With recognition of the stepwise progression of lymph node metastasis (LNM) originating from the central (level VI) to the lateral compartment (levels II-V), a growing number of surgeons are advocating routine prophylactic central neck dissection (pCND) at the time of the total thyroidectomy. ${ }^{3}$ Although the role of pCND has remained controversial because there is little evidence to suggest that it improves long-term outcomes when compared to no pCND, analysis of short-term markers (such as stimulated thyroglobulin) (sTg) seems to suggest pCND may improve short-term outcomes. ${ }^{4-7}$

Because patients with clinically apparent or palpable LNM in the lateral compartment (or N1b) are at significantly higher risk of developing LRR and distant metastases when compared to those with clinically inapparent LNM, size of LNM is believed to be a prognostic factor in patients with PTC. ${ }^{8-14}$ However, to our knowledge, no study has specifically addressed the significance 
of size of central LNM (CLNM) in patients who routinely undergo pCND. For patients who undergo pCND after total thyroidectomy, the incidence of histologically proven CLNM ranged 40-50\%. ${ }^{4-7}$ However, because these CLNM are often small in size and may only be seen under high-power magnification, their clinical significance have been questioned. ${ }^{12,13}$ A recent study reported a series of 72 patients who underwent a pCND and found that none had CLNM $\geq 2 \mathrm{~mm}$ in size (macrometastasis or pN1mac), whereas only 18 patients $(25.0 \%)$ had CLNM $<2 \mathrm{~mm}$ (micrometastasis or pN1mic) present in the central lymph nodes (CLNs). ${ }^{15}$ We hypothesized that size of CLNM might have a negative impact on prognosis, with larger CLNM leading to poorer prognosis. Postsurgical detectable sTg level (DsTg) after radioiodine (RAI) ablation is a good surrogate for persistent or recurrent disease after total thyroidectomy. ${ }^{16-19}$ Therefore, the present study aimed to evaluate the association between size of CLNM and rate of postablation DsTg in patients who underwent an unilateral pCND with particular emphasis on the incidence and significance of $\mathrm{pN} 1 \mathrm{mic}$ and $\mathrm{pN} 1 \mathrm{mac}$.

\section{PATIENTS AND METHODS}

From 2005 to 2011, a total of 250 consecutive patients with PTC underwent surgery in our institution. All were managed by the same surgical team. Of these, $142(56.8 \%)$ underwent a routine unilateral $\mathrm{pCND}$ at the time of the total thyroidectomy. None had evidence of CLNM preoperatively on ultrasonography or intraoperatively. Patients with concomitant lateral lymph node metastases (N1b) or distant metastases (M1) were excluded. All operations were performed by two endocrine surgeons. The decision for a prophylactic unilateral central neck dissection (pCND) was based on the personal preference of the operating surgeon and not based on tumor size or other tumor characteristics. However, there was a tendency to perform more pCND in the latter part of the study period. During the study period, all resected specimens were examined by the same group of pathologists in our institution by a standardized technique. Specimens containing $<3$ CLNs retrieved during pCND $(n=38)$ were excluded to reduce the possibility of nodal under-staging. ${ }^{20}$ Also, patients with anti-thyroglobulin (Tg) autoantibody titer of $>400(n=10)$ were excluded. Therefore, a total of 94 patients were eligible for analysis. Of these, 40 (42.6\%) had no demonstrable LNM on hematoxylin and eosin stain (or pN0) (group I), whereas $54(57.4 \%$ ) had $\geq 1$ demonstrable metastatic focus in CLN on hematoxylin and eosin stain. To measure the size of the CLNM, the relevant slides were retrieved and reexamined by two independent pathologists (A.H.T., T.W.S.). All metastatic foci were measured to the nearest tenth of a millimeter with a built-in computerized measurement program on the microscope (Nikon Eclipse E600). For patients with multiple metastatic foci, only the largest microscopic focus was recorded. To further ensure that the study only evaluated the significance of size of CLNM in the prophylactic setting, 5 patients with CLNM $\geq 1 \mathrm{~cm}$ were excluded because they could not be considered as prophylactic. To evaluate the association between size of CLNM and other clinicopathologic characteristics, patients were categorized into 3 groups: group I (pN0) $(n=40)$, group II (size of CLNM $<2 \mathrm{~mm}$ ) (pN1mic) $(n=20)$ and group III (size of CLN metastasis $\geq 2 \mathrm{~mm})(\mathrm{pN} 1 \mathrm{mac})(n=29)$.

\section{METHODS}

All relevant clinical, laboratory, radiologic and perioperative data were collected prospectively, and follow-up data were regularly updated in a computerized database. The present study protocol was approved by the local institutional review board. Patient clinicopathologic features, sTg and postoperative outcomes were compared between the three groups.

\section{Management of PTC}

Details of surgical treatment, criteria for RAI ablation, postoperative care and follow-up protocol have been described previously. ${ }^{11}$ In brief, total thyroidectomy was the preferred procedure for all patients with a preoperative diagnosis of PTC. A similar extent of unilateral pCND was performed for all patients, regardless of tumor size or extent. ${ }^{6}$ The pCND consisted of the removal of all nodes and fibro-fatty tissue extending vertically from the hyoid bone to the thoracic inlet and laterally from the medial border of common carotid artery to the midline of the trachea. The ipsilateral recurrent laryngeal nerve was mobilized and skeletonized along its entire cervical course. Parathyroid autotransplantation was readily performed. sTg was defined as a $\mathrm{Tg}$ level measured in the presence of thyroid-stimulating hormone (TSH) $>30 \mathrm{mIU} / \mathrm{L}$ either by 4-week thyroxine withdrawal or recombinant TSH injections. The preablation sTg levels were taken approximately 2 months after surgery (at the time of RAI ablation), while the postablation levels were taken approximately 9 months after surgery (6-7 months after RAI ablation or at the time of the whole body scan). Tg autoantibodies were measured at the same time. The decision for RAI ablation was based on the presence of at least one or more risk factors such as tumor size $>1 \mathrm{~cm}$, LNM, age older than 45 years, extrathyroidal extension, macroscopic postoperative residual disease in the neck and distant metastasis, and were not 
dependent on the preablative sTg level. Three giga becquerels or $80 \mathrm{mCi} \mathrm{I}^{131}$ was the standard dose. TSH suppression to $<0.1 \mu \mathrm{g} / \mathrm{L}$ was recommended for high- and intermediate-risk patients.

\section{Follow-up Protocol}

All postsurgical patients were followed up within 4 weeks in a specialized combined oncology clinic. A follow-up visit was conducted at 3-month intervals in the first 2 years, every 6 months in the subsequent 3 years, and annually thereafter. Clinical examination, neck ultrasonography and nonstimulated $\mathrm{Tg}$ level were done during follow-up visits. LRR was confirmed by fine-needle aspiration cytology or histology.

\section{Laboratory Methods}

All postoperative sTg levels were measured at the same laboratory by the same immunometric assay. The assay used was Immulite 2000 (Diagnostic Products, Roche, Los Angeles, CA). This was calibrated against the CRM-457 standard. A sTg level of $\geq 0.5 \mu \mathrm{g} / \mathrm{L}$ was considered a detectable stimulated thyroglobulin (DsTg). Normal reference range was $<0.5-$ $55 \mu \mathrm{g} / \mathrm{L}$, and sensitivity was $<0.2 \mu \mathrm{g} / \mathrm{L}$ ).

\section{Statistical Analyses}

Statistical analyses was performed by $\chi^{2}$ or Fisher's exact test to compare categorical variables, and MannWhitney $U$ or Kruskal-Wallis test was used to compare continuous variables between groups. To evaluate the correlation between two continuous variables, the Spearman rank correlation test was performed. Continuous variables were expressed as medians with ranges. Variables that were significant in the univariate analysis were entered into multivariate analysis. Binary logistic regression analysis with a variable entrance criterion of 0.05 or less was conducted to identify factors associated with postablative DsTg after surgery. Disease-free survivals were estimated by the Kaplan-Meier method and compared with the logrank test (Fig. 1). All statistical analyses were performed by SPSS software, version 18.0 (SPSS, Chicago, IL).

\section{RESULTS}

In our cohort, most were women $(75.3 \%)$ and ethnic Chinese $(89.9 \%)$. The median age at operation was 48.0 (range 8.1-81.3) years, and the median follow-up period was 29.1 (range 9.3-90.1) months. The median primary tumor size was 1.5 (range $0.3-6.0) \mathrm{cm}$, with $8(9.0 \%)$ being microcarcinomas $(<1 \mathrm{~cm})$. The median number of

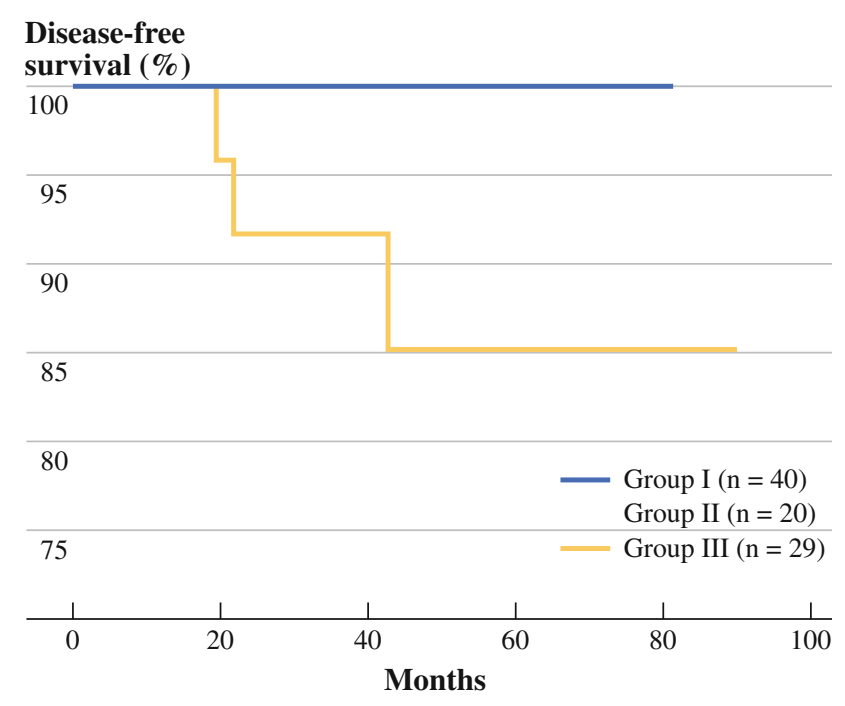

FIG. 1 Cumulative disease-free survival curves of papillary thyroid carcinoma between those with no central lymph node (CLN) metastasis (pN0) (group I), with CLN micrometastases (pN1mic) (group II), and with CLN macrometastases (pN1mac) (group III)

metastatic CLNs was 1 (range 1-18), and the median number of CLNs collected was 6 (range 3-21). The median size of CLNM was 2.6 (range 0.1-6.9) $\mathrm{mm}$.

Table 1 shows a comparison of patient clinicopathologic features, tumor, node, metastasis staging system (TNM) tumor stages, CLN ratio, and metastases, age, completeness of surgery, invasion, and size (MACIS) score between the three groups. The median age at operation was significantly different between the three groups $(p=0.003)$, with group III having the youngest median age of 43.0 years. There was an inverse correlation between age and size of CLNM $(\rho=-0.315, p=0.028)$. Gender, clinical presentation and tumor characteristics such as tumor size, multifocality, capsular invasion, extrathyroidal extension and coexisting thyroiditis were not significantly different between the three groups. Stage of PTC by TNM, MACIS score, postoperative complications and anti-Tg autoantibody titer were also similar between the three groups. Group III had significantly higher number of CLNs retrieved $(p=0.007)$, metastatic CLNs excised $(p<0.001)$ and metastatic CLN ratio $(p=0.010)$ than the other two groups. There was a direct correlation between size of CLNM and number of CLNs retrieved ( $\rho=0.343$, $p=0.016$ ), size of CLNM and number of metastatic CLNs excised $(\rho=0.541, p<0.001)$ and size of CLNM and metastatic CLN ratio $(\rho=0.387, p=0.006)$. Group III also tended to have higher proportion of patients receiving RAI ablation after surgery than groups I and II (96.6 vs. 80.0 and $90.0 \%$, respectively), but this was not statistically significant $(p=0.198)$. In the preablation period the median sTg was similar, whereas in the postablation period the median sTg level was significantly different between 
the three groups $(p=0.012)$. The proportion of DsTg in groups I and II was reduced from 60 to 17.5 and 55 to $15 \%$, respectively, after RAI ablation, whereas the proportion in group III was reduced from 82.8 to $62.1 \%$. At the time of analysis, all patients were alive. In groups I and II, no patient developed LRR, whereas in group III, 2 developed ipsilateral lateral recurrences and 1 developed bilateral lateral recurrence. These three recurrences developed at 19.4, 21.9 and 42.9 months after surgery. All three recurrences had postablation DsTg 9 months after surgery, and their median sTg level was 87.0 (range 9.7-103.0) $\mu \mathrm{g} /$ $\mathrm{L}$. The cumulative disease-free survival in groups I, II and III were not significantly different $(p=0.151)$, and the 5 -and 10-year disease-free survivals in group III were 85.1 and $85.1 \%$, respectively.

Table 2 shows the univariate analysis of clinicopathologic factors for postablation DsTg (i.e., $>0.5 \mu \mathrm{g} / \mathrm{mL}$ ). Age, gender, clinical presentation and most tumor characteristics such as multifocality, capsular invasion, extrathyroidal extension and thyroiditis were similar between those with postablation DsTg and with undetectable sTg. However, relative to those with undetectable sTg, those with postablation DsTg had larger median primary tumor size (2.0 vs. $1.5 \mathrm{~cm}, p=0.018$ ), greater number of metastatic CLNs ( 2 vs. $1, p=0.013$ ), higher CLN ratio (66.7 vs. $20.0 \%, p=0.003)$ and larger size of CLNM ( 4.8 vs. $1.6 \mathrm{~mm}, p<0.001$ ), but the proportion of patients with metastatic CLN (pN1a) was similar between the two groups $(p=0.114)$. When size of CLNM was categorized into types of metastasis ( $\mathrm{pN} 0, \mathrm{pN} 1 \mathrm{mic}$ and $\mathrm{pN} 1 \mathrm{mac}$ ), those with postablation DsTg had a significantly higher proportion of pN1mac (67.7 vs. $10.9 \%)$ but a lower proportion of pN0 (20.6 vs. $60.0 \%)$ and pN1mic (11.8 vs. $29.1 \%)$ than those with undetectable $\operatorname{sTg}(p=0.002)$. There was a significant direct correlation between the size of CLNM and postablation sTg level $(\rho=0.428, p=0.003)$, but such correlation was not observed between size of CLNM and preablation $\operatorname{sTg}(\rho=0.264, p=0.083)$.

Table 3 shows the multivariate analysis of clinicopathologic risk factors for postablative DsTg 9 months after surgery. Variables that were significant in the univariate analysis were entered into the multivariate analysis. The number of metastatic CLNs was not entered because it significantly correlated with the CLN ratio $(\rho=0.896$, $p<0.001$ ), and the CLN ratio was shown to be a more significant factor. ${ }^{21,22}$ Because the size and type of CLNM were similar covariates, they were entered separately into the multivariate analysis with CLN ratio and tumor size. In the multivariate analysis, after adjusting for CLN ratio and tumor size, size of CLNM (expressed as a continuous variable) (odds ratio $1.56,95 \%$ confidence interval $1.09-2.24, p=0.015$ ) was the only independent factor for postablation DsTg. Similarly, when the type of CLNM was entered in the multivariate analysis with CLN ratio and tumor size, relative to $\mathrm{pN} 0$ and $\mathrm{pN} 1 \mathrm{mic}, \mathrm{pN} 1 \mathrm{mac}$ was the only independent risk factor for postablation DsTg (odds ratio 1.76, $95 \%$ confidence interval 1.22-27.70, $p=0.027)$.

\section{DISCUSSION}

Previous studies have found that size of LNM in the lateral compartment is a significant risk factor for LRR, distant metastasis and poor overall survival. ${ }^{8-11,14}$ Sugitani et al. reported that for patients older than 50 years, LNM $\geq 3 \mathrm{~cm}$ was an independent factor for cancer-specific survival from PTC. ${ }^{10}$ Similarly, it was observed that those with clinically apparent or palpable LNM were at significantly higher risk of developing LRR and distant metastases than those with clinically inapparent or nonpalpable $\mathrm{LNM}^{8-13}$ In fact, this was the rationale for performing therapeutic selective neck dissection in the lateral compartment and not prophylactic selective neck dissection in patients with PTC. ${ }^{13}$ However, in comparison to lateral selective neck dissection, pCND is associated with lower surgical morbidity and can be performed within the same incision as the thyroidectomy. ${ }^{23}$ In addition, it provides more information regarding the nodal status as required by the TNM and may lower LRRs. ${ }^{23}$ Several studies confirmed that those who underwent pCND had a significantly lower postsurgical sTg than those without pCND. This perhaps suggests that pCND was able to provide a more oncologically complete resection than thyroidectomy alone by excising micrometastases harbored in the central compartment. ${ }^{4-6}$ This hypothesis was supported by the fact that over $50 \%$ of CLNs resected harbored metastases. ${ }^{4-7}$ However, questions remain regarding the size of these metastases and their importance to patient outcomes. Given that the size of LNM in the lateral compartment is a prognostic factor, we hypothesized that the size of CLNM in pCND would also be a prognostic factor, although this has not yet been demonstrated.

To ensure that our study only evaluated CLNs taken out of pCND, 5 patients with CLNM $\geq 1 \mathrm{~cm}$ were excluded. In our analysis, we found that even when the size of CLNM was limited to $<1 \mathrm{~cm}$, it was an independent factor for postablation DsTg 9 months after surgery. There was a significant direct correlation between the size of CLNM and postablation sTg level $(\rho=0.428, \quad p=0.003)$, although not between size of CLNM and preablation $\mathrm{sTg}$ ( $\rho=0.264, p=0.083)$. When the size of CLNM was correlated with other clinicopathologic characteristics, we observed that the size of CLNM was inversely correlated with age (i.e., younger patients had larger-sized CLNM), but this was directly correlated with greater number of CLNs, metastatic CLNs and higher metastatic CLN ratio. This latter association implied that larger-sized CLNM 
TABLE 1 A comparison of patient characteristics between group I, II and III

Boldface signifies $p$-value $<0.05$ or statistically significant

Continuous variables are expressed as median (range); categorical variables are expressed as $n(\%)$

PTC papillary thyroid carcinoma, TNM 6th edition of the tumor, node and metastasis staging system, $C L N$ central lymph node, MACIS metastases, age, completeness of surgery, invasion, and size, TSH thyroidstimulating hormone, DsTg detectable stimulated thyroglobulin $(>0.5 \mu \mathrm{g} / \mathrm{L})$

${ }^{\mathrm{a}} \mathrm{CLN}$ ratio $=$ (no. of metastatic CLNs/no. of CLNs retrieved) $\times 100$

${ }^{b}$ Calculated on the basis of number of nerves at risk

\begin{tabular}{|c|c|c|c|c|}
\hline Characteristic & Group I $(n=40)$ & Group II $(n=20)$ & Group III $(n=29)$ & $p$-value \\
\hline Age at operation (y) & $52(17-81)$ & $48(8-73)$ & $43(21-67)$ & 0.003 \\
\hline Sex & & & & 0.814 \\
\hline Male & $9(22.5)$ & $6(30.0)$ & $7(24.1)$ & \\
\hline Female & $31(77.5)$ & $14(70.0)$ & $22(75.9)$ & \\
\hline Clinical presentation & & & & 0.310 \\
\hline Incidental & $13(32.5)$ & $9(45.0)$ & $7(24.1)$ & \\
\hline Symptomatic & $27(67.5)$ & $11(55.0)$ & $22(75.9)$ & \\
\hline \multicolumn{5}{|l|}{ Tumor characteristics } \\
\hline Tumor size $(\mathrm{cm})$ & $1.5(0.3-3.8)$ & $1.5(0.3-4.0)$ & $2.0(0.5-6.0)$ & 0.266 \\
\hline Multifocality & $15(37.5)$ & $6(30.0)$ & $10(34.5)$ & 0.901 \\
\hline Capsular invasion & $10(25.0)$ & $6(30.0)$ & $5(17.2)$ & 0.509 \\
\hline Extrathyroidal extension & $14(56.0)$ & $4(20.0)$ & $6(20.7)$ & 0.331 \\
\hline Coexisting thyroiditis & $8(20.0)$ & $4(20.0)$ & $7(24.1)$ & 0.378 \\
\hline Stage of PTC by TNM & & & & 0.198 \\
\hline Stage I/II & $20(50.0)$ & $6(30.0)$ & $16(55.2)$ & \\
\hline Stage III/IV & $20(50.0)$ & $14(70.0)$ & $13(44.8)$ & \\
\hline Size of CLN metastasis (mm) & - & $1.1(0.1-1.8)$ & $4.2(2.0-6.9)$ & $<\mathbf{0 . 0 0 1}$ \\
\hline No. of CLNs retrieved & $4(3-18)$ & $5.5(3-9)$ & $8(3-21)$ & 0.007 \\
\hline No. of metastatic CLNs excised & - & $1(1-6)$ & $4(1-18)$ & $<\mathbf{0 . 0 0 1}$ \\
\hline Metastatic CLN ratio $(\%)^{\mathrm{a}}$ & - & $29.2(12.5-100)$ & $69.2(12.5-100)$ & 0.010 \\
\hline MACIS score & $5.2(3.2-8.0)$ & $4.9(3.3-12.0)$ & $4.3(3.2-8.9)$ & 0.094 \\
\hline Postoperative hypocalcemia & & & & 0.739 \\
\hline Temporary & $5(12.5)$ & $4(20.0)$ & $6(20.7)$ & \\
\hline Permanent & $1(2.5)$ & $0(0.0)$ & $1(3.4)$ & \\
\hline Postoperative vocal cord palsy ${ }^{b}$ & & & & 0.348 \\
\hline Temporary & $0(0.0)$ & $1(2.5)$ & $2(3.4)$ & \\
\hline Permanent & $0(0.0)$ & $1(2.5)$ & $2(3.4)$ & \\
\hline Radioiodine ablation & $32(80.0)$ & $18(90.0)$ & $28(96.6)$ & 0.198 \\
\hline Anti-Tg antibody (titers) & & & & 0.399 \\
\hline$\leq 99$ & $33(82.5)$ & $15(75.0)$ & $26(89.7)$ & \\
\hline $100-400$ & $7(17.5)$ & $5(25.0)$ & $3(10.3)$ & \\
\hline \multicolumn{5}{|l|}{ Preablation period } \\
\hline TSH level (mIU/L) & $62(34-339)$ & $48(35-226)$ & $57(37-250)$ & 0.826 \\
\hline Stimulated Tg level $(\mu \mathrm{g} / \mathrm{L})$ & $1.2(<0.5-15.0)$ & $0.9(<0.5-114)$ & $3.4(<0.5-196)$ & 0.141 \\
\hline No. of DsTg & $24(60.0)$ & $11(55.0)$ & $24(82.8)$ & 0.070 \\
\hline \multicolumn{5}{|l|}{ Postablation period } \\
\hline TSH level (mIU/L) & $87(65-253)$ & $91(86-146)$ & $68(39-229)$ & 0.595 \\
\hline Stimulated Tg level $(\mu \mathrm{g} / \mathrm{L})$ & $<0.5(<0.5-4.7)$ & $<0.5(<0.5-9.2)$ & $0.8(<0.5-110)$ & 0.012 \\
\hline No. of DsTg & $7(17.5)$ & $3(15.0)$ & $18(62.1)$ & 0.001 \\
\hline Locoregional recurrence & $0(0.0)$ & $0(0.0)$ & $3(10.3)$ & 0.157 \\
\hline
\end{tabular}

statistical significance; this may imply that $\mathrm{pN} 1 \mathrm{mic}$ did not have the same significance level as pN1mac on postablation DsTg.

However, because the size of CLNM is a histopathologic finding, its clinical application could only be limited to the postsurgical management. Nevertheless, one possible application would be to use it as a factor for deciding on the dose of RAI ablation. Because most patients with either is worth noting that the odds ratio for $\mathrm{pN} 1 \mathrm{mic}$ did not reach 
TABLE 2 Univariate analysis of clinicopathologic risk factors for detectable postablation stimulated thyroglobulin level $(>0.5 \mu \mathrm{g} / \mathrm{mL})$

\begin{tabular}{|c|c|c|c|}
\hline Characteristic & $\begin{array}{l}\text { Detectable } \\
\text { postablation sTg } \\
(n=34)\end{array}$ & $\begin{array}{l}\text { Undetectable } \\
\text { postablation sTg } \\
(n=55)\end{array}$ & $p$-value \\
\hline $\begin{array}{l}\text { Age at operation } \\
\text { (years) }\end{array}$ & $43(21-78)$ & $50(17-81)$ & 0.065 \\
\hline Sex & & & 0.881 \\
\hline Male & $9(26.5)$ & $13(23.6)$ & \\
\hline Female & $25(73.5)$ & $42(76.4)$ & \\
\hline Clinical presentation & & & 0.063 \\
\hline Incidental & $5(14.7)$ & $24(43.6)$ & \\
\hline Symptomatic & $29(85.3)$ & $31(56.4)$ & \\
\hline \multicolumn{4}{|l|}{ Tumor characteristics } \\
\hline Tumor size (cm) & $2.0(0.3-6.0)$ & $1.5(0.5-4.0)$ & 0.018 \\
\hline Multifocality & $16(47.1)$ & $15(27.3)$ & 0.125 \\
\hline Capsular invasion & $11(32.4)$ & $10(18.2)$ & 0.390 \\
\hline $\begin{array}{l}\text { Extrathyroidal } \\
\text { extension }\end{array}$ & $15(44.1)$ & $9(16.4)$ & 0.445 \\
\hline $\begin{array}{c}\text { Coexisting } \\
\text { thyroiditis }\end{array}$ & $6(17.6)$ & $13(23.6)$ & 0.299 \\
\hline $\begin{array}{l}\text { Stage of PTC } \\
\text { byTNM }\end{array}$ & & & 0.217 \\
\hline Stage I/II & $19(55.9)$ & $23(41.8)$ & \\
\hline Stage III/IV & $15(44.1)$ & $32(58.2)$ & \\
\hline $\begin{array}{l}\text { No. of CLNs } \\
\text { retrieved }\end{array}$ & $6(3-21)$ & $6(4-18)$ & 0.814 \\
\hline $\begin{array}{l}\text { No. of metastatic } \\
\text { CLNs excised }\end{array}$ & $2(0-18)$ & $1(0-9)$ & 0.013 \\
\hline $\begin{array}{l}\text { Metastatic CLN } \\
\text { ratio }(\%)^{\mathrm{b}}\end{array}$ & $66.7(0-100)$ & $20(0-100)$ & 0.003 \\
\hline $\begin{array}{l}\text { Metastatic CLN } \\
\quad(\mathrm{pN} 1 \mathrm{a})\end{array}$ & $20(58.8)$ & $29(52.7)$ & 0.114 \\
\hline $\begin{array}{l}\text { Size of CLN } \\
\text { metastasis (mm) }\end{array}$ & $4.8(0.3-6.9)$ & $1.6(0.1-6.1)$ & $<\mathbf{0 . 0 0 1}$ \\
\hline $\begin{array}{l}\text { Type of CLN } \\
\text { metastasis }\end{array}$ & & & 0.002 \\
\hline pNO & $7(20.6)$ & $33(60.0)$ & \\
\hline pN1mic & $4(11.8)$ & $16(29.1)$ & \\
\hline pN1mac & $23(67.6)$ & $6(10.9)$ & \\
\hline
\end{tabular}

Boldface signifies $p$-value $<0.05$ or statistically significant

$s T g$ stimulated thyroglobulin, PTC papillary thyroid carcinoma, $T N M$ 6th edition of the tumor, node and metastasis staging system, $C L N$ central lymph node, $p N O$ no central lymph node metastasis, pN1mic micrometastasis (size $\leq 2 \mathrm{~mm}$ ), pN1mac macrometastasis (size $>2 \mathrm{~mm}$ )

${ }^{a}$ Continuous variables are expressed as median (range); categorical variables are expressed as $n(\%)$

${ }^{\mathrm{b}}$ Metastatic CLN ratio $=$ (no. of metastatic CLNs/no. of CLNs retrieved) $\times 100$

pN1mic or pN1mac would receive at least one ablative dose, it would be rational to have those with pN1mac receiving a higher dose of ablation (instead of the standard dose of $3 \mathrm{GBq}$ ) than those with pN1mic or pN0 when RAI
TABLE 3 Multivariate analysis of clinicopathologic risk factors for postablative detectable stimulated thyroglobulin 9 months after surgery

\begin{tabular}{llll}
\hline Covariate & $\beta$ & $\begin{array}{l}\text { Odds ratio (95\% confidence } \\
\text { Coefficient }\end{array}$ & $p$-value \\
\hline $\begin{array}{l}\text { Metastatic CLN } \\
\text { ratio }^{\mathrm{a}}\end{array}$ & 0.014 & $1.014(0.990-1.039)$ & 0.251 \\
$\begin{array}{l}\text { Primary tumor } \\
\text { size }\end{array}$ & 0.389 & $1.476(0.848-2.568)$ & 0.168 \\
$\begin{array}{l}\text { Type of CLN metastasis } \\
\text { pN0 }\end{array}$ & 1 & \\
pN1mic & 0.949 & $2.583(0.351-19.000)$ & 0.351 \\
pN1mac & 1.760 & $5.814(1.221-27.697)$ & $\mathbf{0 . 0 2 7}$ \\
\hline
\end{tabular}

Boldface signifies $p$-value $<0.05$ or statistically significant

$C L N$ central lymph node, $p N O$ no CLN metastasis, pN1mic micrometastasis (size $\leq 2 \mathrm{~mm}$ ), pN1mac macrometastasis (size $>2 \mathrm{~mm}$ )

${ }^{\text {a }}$ Metastatic CLN ratio $=($ no. of metastatic CLNs/no. of CLNs retrieved) $\times 100$

b When size of CLN metastasis was entered instead, the odds ratio became 1.564 (95\% confidence interval 1.092-2.241), $p=0.015$

ablation is indicated. In our study, it was interesting to observe that up to $42.5 \%$ of patients with either pN0 or pN1mic became athyroglobulinemic after $3 \mathrm{GBq}$ of RAI ablation, whereas only $20.7 \%$ in the pN1mac group became athyroglobulinemic. This means that the latter group might perhaps benefit from a higher dose of RAI ablation if athyroglobulinemia is the aim. If future studies could confirm the size of CLNM as an independent factor for outcomes, reporting the size of CLNM in the histopathology reports could become important.

Despite these findings, we acknowledge shortcomings with our study, including the relatively small number of patients within each group and potential biases in the selection for $\mathrm{pCND}$. As a measure of outcome, some would argue that a sTg of $0.5 \mu \mathrm{g} / \mathrm{L}$ might be set too low and a level taken at 9 months after surgery might be too early. However, as shown previously, a sTg of $<0.5 \mu \mathrm{g} / \mathrm{L}$ at 1 year had a $>98 \%$ likelihood of identifying patients completely free of disease at follow-up, and a sTg level taken at 1 year after surgery was as good as a level taken $2-3$ years after surgery. ${ }^{24,25}$ Therefore, we believe sTg $<0.5 \mu \mathrm{g} / \mathrm{L}$ at 9 months after surgery is a reasonable surrogate for future recurrences. Although not statistically significant, it is worth noting that of the 3 LRR, all belonged to the pN1mac group and had postablation DsTg with a median sTg level of 87.0 (range 9.7-103.0) $\mu \mathrm{g} / \mathrm{L}$. In contrast, none of the patients in $\mathrm{pN} 0$ or pN1mic developed recurrences.

In conclusion, pN1mic accounted for $20(22.5 \%)$ of 89 patients undergoing pCND. Larger-sized CLNM was significantly associated with younger age, greater number of CLN retrieved, greater number of metastatic CLN excised, higher metastatic CLN ratio and higher postablation sTg 
level. Tumor size, metastatic CLN ratio and size of CLNM were significantly associated with postablation DsTg, but only size of CLNM turned out to be an independent factor for postablation DsTg. Patients with pN1mac were almost 6 times more likely to have postablation DsTg than those with $\mathrm{pN} 0$ or $\mathrm{pN} 1 \mathrm{mic}$.

OPEN ACCESS This article is distributed under the terms of the Creative Commons Attribution License which permits any use, distribution, and reproduction in any medium, provided the original author(s) and the source are credited.

\section{REFERENCES}

1. Cancer incidence and mortality in Hong Kong, 1983-2006. Hong Kong Cancer Registry, Hong Kong. http://www3.ha.org.hk/ cancereg/e_stat.asp. Accessed 15 Dec 2011.

2. Lang BH, Lo CY, Chan WF, Lam KY, Wan KY. Prognostic factors in papillary and follicular thyroid carcinoma: implications for cancer staging. Ann Surg Oncol. 2007;14:730-8.

3. Machens A, Hauptmann S, Dralle H. Lymph node dissection in the lateral neck for completion in central node-positive papillary thyroid cancer. Surgery. 2009;145:176-81.

4. Sywak M, Cornford L, Roach P, Stalberg P, Sidhu S, Delbridge L. Routine ipsilateral level VI lymphadenectomy reduces postoperative thyroglobulin levels in papillary thyroid carcinoma. Surgery. 2006;140:1000-7.

5. So YK, Seo MY, Son YI. Prophylactic central lymph nodes dissection for clinically node-negative papillary thyroid microcarcinoma: influence on serum thyroglobulin level, recurrence rate, and postoperative complication. Surgery. 2012;151:192-8.

6. Lang BH, Wong KP, Wan KY, Lo CY. Impact of routine unilateral central neck dissection on preablative and postablative stimulated thyroglobulin levels after total thyroidectomy in papillary thyroid carcinoma. Ann Surg Oncol. 2012;19:60-7.

7. Hughes DT, White ML, Miller BS, Gauger PG, Burney RE, Doherty GM. Influence of prophylactic central lymph node dissection on postoperative thyroglobulin levels and radioiodine treatment in papillary thyroid cancer. Surgery. 2010;148:1100-6.

8. Bardet S, Malville E, Rame JP, et al. Macroscopic lymph-node involvement and neck dissection predict lymph-node recurrence in papillary thyroid carcinoma. Eur J Endocrinol. 2008;158: 551-60.

9. Mercante G, Frasoldati A, Pedroni C, et al. Prognostic factors affecting neck lymph node recurrence and distant metastasis in papillary microcarcinoma of the thyroid: results of a study in 445 patients. Thyroid. 2009;19:707-16.

10. Sugitani I, Kasai N, Fujimoto Y, Yanagisawa A. A novel classification system for patients with PTC: addition of the new variables of large $(3 \mathrm{~cm}$ or greater) nodal metastases and reclassification during the follow-up period. Surgery. 2004;135: $139-48$.

11. Lang BH, Lo CY, Chan WF, Lam KY, Wan KY. Staging systems for papillary thyroid carcinoma: a review and comparison. Ann Surg. 2007;245:366-78.
12. Cranshaw IM, Carnaille B. Micrometastases in thyroid cancer. An important finding? Surg Oncol. 2008;17:253-8.

13. Caron NR, Clark OH. Papillary thyroid cancer: surgical management of lymph node metastases. Curr Treat Options Oncol. 2005;6:311-22.

14. Leboulleux S, Rubino C, Baudin E, et al. Prognostic factors for persistent or recurrent disease of papillary thyroid carcinoma with neck lymph node metastases and/or tumor extension beyond the thyroid capsule at initial diagnosis. J Clin Endocrinol Metab. 2005;90:5723-9.

15. Teixeira G, Teixeira T, Gubert F, Chikota H, Tufano R. The incidence of central neck micrometastatic disease in patients with papillary thyroid cancer staged preoperatively and intraoperatively as NO. Surgery. 2011;150:1161-7.

16. Cooper DS, Doherty GM, Hauger BR, et al. Revised American Thyroid Association management guidelines for patients with thyroid nodules and differentiated thyroid cancer. Thyroid. 2009;19:1167-214.

17. Kim TY, Kim WB, Kim ES, et al. Serum thyroglobulin levels at the time of 131I remnant ablation just after thyroidectomy are useful for early prediction of clinical recurrence in low-risk patients with differentiated thyroid carcinoma. J Clin Endocrinol Metab. 2005;90:1440-5.

18. Heemstra KA, Liu YY, Stokkel M, et al. Serum thyroglobulin concentrations predict disease-free remission and death in differentiated thyroid carcinoma. Clin Endocrinol (Oxf). 2007;66:58-64.

19. Giovanella L, Ceriani L, Suriano S, Ghelfo A, Maffioli M. Thyroglobulin measurement before rh-TSH-aided 131I ablation in detecting metastases form differentiated thyroid carcinoma. Clin Endocrinol (Oxf). 2008;69:659-63.

20. Lang B, Lo CY, Chan WF, Lam KY, Wan KY. Restaging of differentiated thyroid carcinoma by the sixth edition AJCC/UICC TNM staging system: stage migration and predictability. Ann Surg Oncol. 2007;14:1551-9.

21. Lang BH, Wong KP, Wan KY, Lo CY. Significance of metastatic lymph node ratio on stimulated thyroglobulin levels in papillary thyroid carcinoma after prophylactic unilateral central neck dissection. Ann Surg Oncol. 2012;19:1257-63.

22. Takada H, Kikumori T, Imai T, Sawaki M, Shibata A, Kiuchi T. Patterns of lymph node metastases in papillary thyroid carcinoma: results from consecutive bilateral cervical lymph node dissection. World J Surg. 2011;35:1560-6.

23. Wong KP, Lang BH. The role of prophylactic central neck dissection in differentiated thyroid carcinoma: issues and controversies. J Oncol. 2011;2011:127929.

24. Kloos RT, Mazzaferri EL. A single recombinant human thyrotrophin-stimulated serum thyroglobulin measurement predicts differentiated thyroid carcinoma metastases three to five years later. J Clin Endocrinol Metab. 2005;90:5047-57.

25. Castagna MG, Brilli L, Pilli T, et al. Limited value of repeat recombinant thyrotropin (rhTSH)-stimulated thyroglobulin testing in differentiated thyroid carcinoma patients with previous negative rhTSH stimulated thyroglobulin and undetectable basal serum thyroglobulin levels. J Clin Endocrinol Metab. 2008; 93:76-81. 